\title{
Caractères du système protéolytique de Geotrichum candidum
}

\author{
par \\ Micheline GUEGUEN* et J. LENOIR \\ Laboratoire de Recherch? de la Chaire de Technologie (I.N.R.A.) \\ Institut National Agronomique Paris-Grignon \\ 78850 Thiverval-Grignon
}

\section{INTRODUCTION}

Geotrichum candidum Link est une moisissure présente à la surface de nombreux fromages. Elle joue un rôle important dans la couverture du "Pont-l'Evêque » et du " Livarot " (Hurel et Mocquot, 1947). Par sa présence, elle favorise l'implantation de Penicillium caseicolum sur le Camembert (Sansonetti, 1930), mais au contraire, d'après les observations des professionnels, elle tend plutôt à limiter le développement des Mucor sp, responsables de l'accident du " poil de chat ". Son implantation est recherchée sur les fromages à pâte pressée des types Saint-Nectaire et Tome de Savoie. Elle forme à la surface de ces fromages un feutrage blanc-gris, et il a été, là aussi, constaté qu'elle assure une protection contre le développement de moisissures indésirables (Dale et Guillot, 1971 ; Dale, 1972 ; Delespaul et al., 1973 ; Gueguen et al., 1974).

Outre ce rôle protecteur, Geotrichum candidum peut contribuer à l'affinage des fromages par l'action de ses enzymes sur les constituants du caillé. Le système lipolytique de l'espèce a fait l'objet de nombreux travaux et il est assez bien caractérisé (Nelson, 1952, 1953 ; Dluzewski, 1963 ; Carini et al., 1970 ; Tsujisaka et al., 1973 ; Franzke et al., 1973) ; en revanche, son système protéolytique reste mal connu.

Après avoir déterminé l'aptitude de l'espèce à la protéolyse sur une collection de 86 souches isolées de divers fromages, et noté des variations importantes d'une souche à l'autre (Gueguen et Lenoir, 1975), il nous a paru intéressant de préciser les caractères du système

* Adresse actuelle : Laboratoire de Microbiologie, Université de Caen, rue du Gaillon - 14000 Caen. 
protéolytique. Portant sur plusieurs souches, l'étude doit permettre de déterminer si les différences d'activité s'accompagnent ou non de différences dans la nature du système, et, en même temps, de s'assurer de ses possibilités d'intervention au cours de l'affinage des pâtes fromagères.

\section{PROTOCOLE EXPERIMENTAL}

\section{I.1. Sélection des souches}

Six souches d'activités différentes ont été retenues en vue de la précaractérisation du système protéolytique : G. 36 et G. 116 de faible activité, G. 59 et G. 819 qui présentent une activité moyenne, G. 828 et G. 830 qui ont une forte activité protéolytique. Les trois premières souches ont l'aspect levuriforme typique de l'espèce, les trois autres sont légèrement duveteuses. Une souche appartenant à l'espèce G. gracile (D. 72) et présentant une activité protéolytique moyenne a été également étudiée.

\section{I.2. Méthodes}

\section{I.2.1. Conditions de culture}

Après une préculture de $8 \mathrm{j}$ à $23^{\circ} \mathrm{C}$ sur milieu Czapek-trypticase (CT) gélosé, les souches sont cultivées $11 \mathrm{j}$ à $23^{\circ} \mathrm{C}$ en milieu $\mathrm{CT}$ liquide non agité. Le milieu CT* ${ }^{*}$ dérivé du milieu Czapek-Dox (Thom et Raper, 1945), est maintenu à $\mathrm{pH} 5,5$ à l'aide de tampon tris-maléate $0,05 \mathrm{M}$ et réparti en erlenmeyers de $150 \mathrm{ml}$ à raison de $40 \mathrm{ml}$ par fiole.

\section{I.2.2. Conditions de détermination de l'activité protéolytique}

Ces conditions ont été décrites antérieurement (Gueguen et Lenoir, 1975 a). Le filtrat de culture, ajusté à $\mathrm{pH}$ 6,0, constitue la préparation enzymatique extracellulaire, le mycélium broyé en présence de tampon phosphate $0,1 \mathrm{M} \mathrm{pH} \mathrm{6,0} \mathrm{la} \mathrm{préparation} \mathrm{intracellulaire.} \mathrm{Les} \mathrm{prépa-}$ rations enzymatiques $(1 \mathrm{ml})$ sont ajoutées au mélange caséinate de sodium 2,5 ml, tampon mixte acétate-borate-phosphate $0,25 \mathrm{M} \mathrm{pH} \mathrm{6,0}$ $0,5 \mathrm{ml}$, eau distillée $1 \mathrm{ml}$, ajusté au $\mathrm{pH}$ voulu. Après incubation $1 \mathrm{~h}$ à la température choisie, le degré de protéolyse est déterminé, sur les filtrats trichloracétiques à 2 et 12 p. 100, par dosage de la tyrosine avec le réactif de Folin, selon la méthode de Anson (1938), et par dosage des groupements $\mathrm{NH}_{2}$ libres par réaction à la ninhydrine. Tous les essais sont réalisés en double.

\footnotetext{
* Composition du milieu Czapek-trypticase : glucose, $30 \mathrm{~g}$; trypticase, $10 \mathrm{~g}$; $\mathrm{KH}_{2} \mathrm{PO}_{4}, 1 \mathrm{~g} ; \mathrm{KCl}, 0,5 \mathrm{~g} ; \mathrm{MgSO}_{4}, 7 \mathrm{H}_{2} \mathrm{O}, 0,5 \mathrm{~g} ; \mathrm{FeSO}_{4}, 7 \mathrm{H}_{2} \mathrm{O}, 0,01 \mathrm{~g}$; eau q.s.p. $1000 \mathrm{ml}$.
} 


\section{I.2.3. Conditions d'étude des facteurs de l'activité protéolytique}

Une expérience préliminaire, réalisée sur les souches G. 819 et D. 72, a permis de constater que, dans l'intervalle de $\mathrm{pH}$ 3-11, l'activité protéolytique était nulle ou négligeable en-dessous de $\mathrm{pH}$ 4,0 et audessus de $\mathrm{pH} 8,0$. La gamme de $\mathrm{pH}$ retenue pour déterminer le $\mathrm{pH}$ optimal d'activité est donc 4-8.

Pour l'étude de la stabilité en fonction du $\mathrm{pH}$, les préparations enzymatiques sont amenées au pH voulu dans l'intervalle 3-11 et conservées à ce $\mathrm{pH}$ pendant $1 \mathrm{~h}$ à $40^{\circ} \mathrm{C}$. L'activité résiduelle est déterminée par digestion à $\mathrm{pH} 6,0,1 \mathrm{~h}$ à $55^{\circ} \mathrm{C}$ pour l'activité extracellulaire, $1 \mathrm{~h}$ à $50^{\circ} \mathrm{C}$ pour l'activité intracellulaire.

La température optimale d'action est recherchée par digestion à $\mathrm{pH} 6,0$ dans l'intervalle de $5-60^{\circ} \mathrm{C}$ pour l'activité extracellulaire, $25-60^{\circ} \mathrm{C}$ pour l'activité intracellulaire, les temps de digestion étant de $23 \mathrm{~h}$ dans la plage $5-25^{\circ} \mathrm{C}, 1 \mathrm{~h}$ dans la plage $25-60^{\circ} \mathrm{C}$.

Pour la détermination de la stabilité thermique, les préparations enzymatiques, réglées à $\mathrm{pH} 6,0$, sont portées à $60^{\circ} \mathrm{C}$ pendant des temps de 1 à $30 \mathrm{mn}$ dans des tubes de verre de $5 \mathrm{~mm}$ de diamètre ; l'activité résiduelle est déterminée comme indiqué ci-dessus.

Dans les essais de stabilité en fonction de la température, les préparations sont maintenues pendant $1 \mathrm{~h}$ à $\mathrm{pH} 6,0$ à une température variant de 5 à $60^{\circ} \mathrm{C}$ avant d'être ajoutées au substrat.

\section{RESULTATS ET DISCUSSION}

\section{II.1. Activité protéolytique extracellulaire}

L'activité extracellulaire est maximale aux valeurs de $\mathrm{pH}$ comprises entre 5,5 et 6,0 (fig. 1 a). Dans cet intervalle, les préparations obtenues à partir de $G$. candidum sont peu sensibles aux variations de $\mathrm{pH}$; en revanche, celle de $G$. gracile présente un optimum net au voisinage de $\mathrm{pH} 6,0$ et elle perd environ 30 p. 100 de son activité pour une variation de 0,5 unité $\mathrm{pH}$ autour de l'optimum.

Si l'on établit les rapports des teneurs en produits solubles en acide trichloracétique à 12 p. 100 / produits solubles en acide trichloracétique à 2 p. 100 (tyr. 12 / tyr. 2), des valeurs de 0,40 à 0,70 sont notées. Fortes aux $\mathrm{pH}$ acides, elles tendent à diminuer près de la neutralité. Au voisinage du $\mathrm{pH}$ optimal, les produits libérés sont donc de poids moléculaire plus élevé qu'aux $\mathrm{pH}$ de $4-5$. Les variations du rapport groupements $\mathrm{NH}_{2}$ libres / produits solubles, ou Leucine / Tyrosine (Leu / Tyr), qui diminue avec l'augmentation du $\mathrm{pH}$, confirment cette observation. 
Activité protéolytique

a

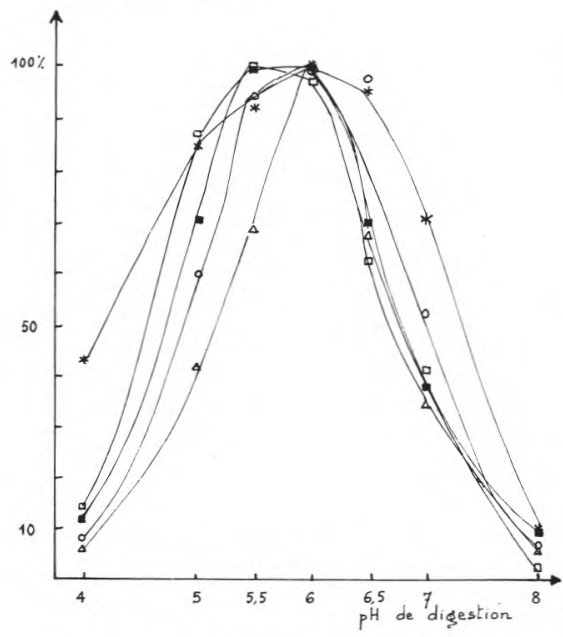

Activité protéolytique

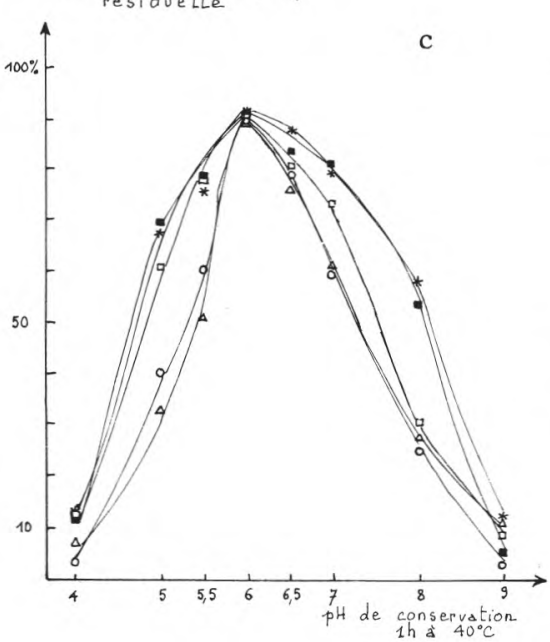

Activité Protéolytique
en \% de l'optimum.

b

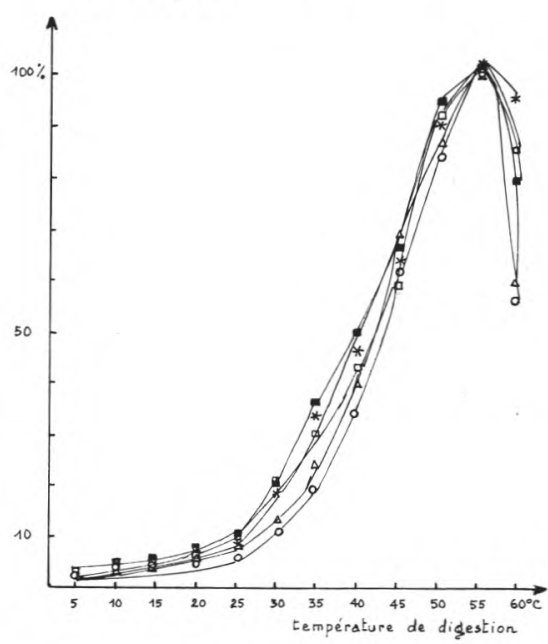

Log. de L'Activité

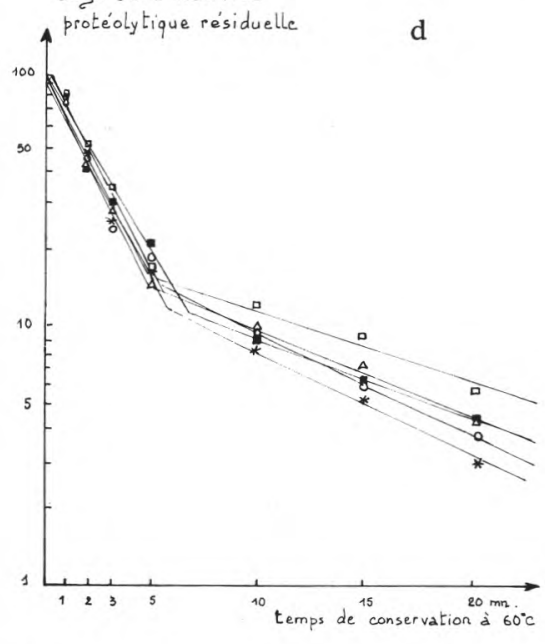

fig. 1

Caractères de l'activité protéolytique extracellulaire

Figure 1 a : Influence du pH sur l'activité.

- En abcisses : $\mathrm{pH}$ du mélange de digestion.

- En ordonnées : activité protéolytique exprimée en p.100 de l'activité optimale.

Figure $1 \mathrm{~b}$ : Influence de la température sur l'activité.

- En abcisses : température du mélange de digestion.

- En ordonnées : cf. fig. 1 a. 
Figure 1 c : Influence du pH sur la stabilité.

- En abcisses : $\mathrm{pH}$ de conservation de la préparation enzymatique.

- En ordonnées : activité protéolytique résiduelle exprim.ée en p. 100 de l'activité initiale.

Figure $1 \mathrm{~d}$ : Stabilité thermique.

- En abcisses : temps de conservation des préparations à $60^{\circ} \mathrm{C}$ à $\mathrm{pH} 6,0$.

- En ordonnées : log de l'activité protéolytique résiduelle exprimée en p. 100 de l'activité initiale.

Chez les souches G. 36 et G. 116, la production d'enzymes extracellulaires est très faible, et les variations d'activité, exprimées en p. 100 de l'activité maximale, ne sont pas suffisamment significatives pour être prises en considération.

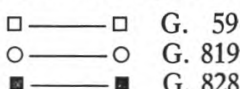
G. 59
G. 828

G. 830

D. 72

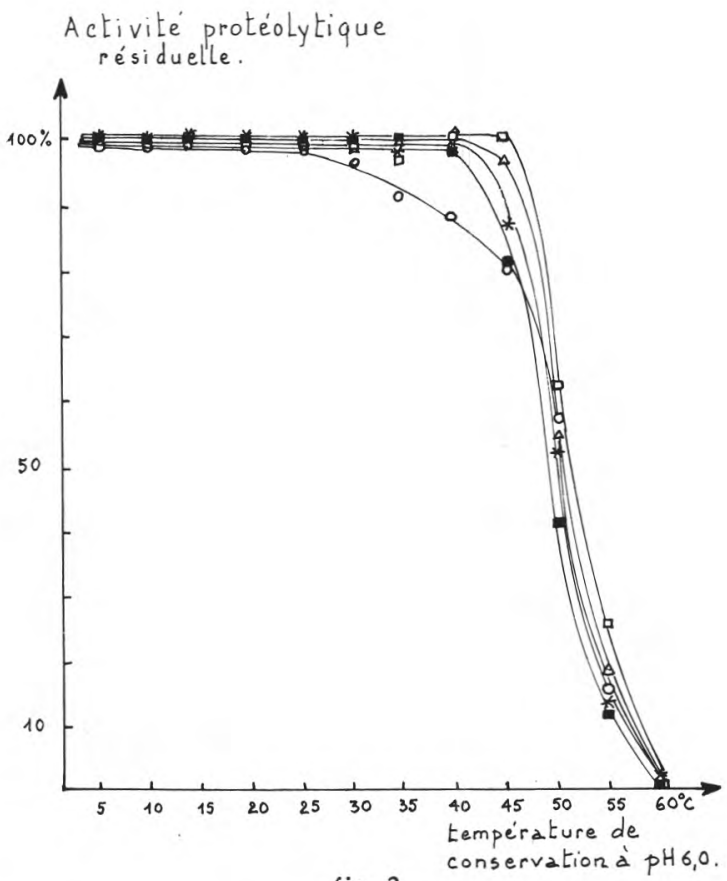

fig. 2

Influence de la température sur la stabilité du système protéolytique extracellulaire

- En abcisses : température de conservation des prépations réglées à $\mathrm{pH}$ 6,0.

- En ordonnées : activité protéolytique résiduelle exprimée en p. 100 de l'activité initiale. 
La température optimale d'activité se situe à $55^{\circ} \mathrm{C}$. Cet optimum est bien marqué ; à $40^{\circ} \mathrm{C}$ l'activité n'est plus que de 35 à $50 \mathrm{p} .100 \mathrm{de}$ l'activité maximale ; à $60^{\circ} \mathrm{C}$, elle atteint selon les souches 55 à 85 p. 100 de celle-ci (fig. 1 b). Les valeurs des rapports Leu / Tyr et Tyr 12 / Tyr 2 passent par un maximum entre $20^{\circ} \mathrm{C}$ et $30^{\circ} \mathrm{C}$ puis diminuent progressivement avec l'accroissement de la température. Le poids moléculaire des produits solubles, augmente donc quand la température de digestion passe de $30^{\circ} \mathrm{C}$ à $60^{\circ} \mathrm{C}$.

Le système extracellulaire présente un maximum de stabilité dans la zone de son $\mathrm{pH}$ optimal, c'est-à-dire au voisinage de $\mathrm{pH}$ 6,0 (fig. $1 \mathrm{c}$ ). Il apparaît très sensible aux variations de $\mathrm{pH}$ et il est pratiquement inactivé aux pH inférieurs à 4,0 ou supérieurs à 9,0 .

La stabilité en fonction de la température est représentée sur la fig. 2. Elle commence à diminuer à partir de $40^{\circ} \mathrm{C}-45^{\circ} \mathrm{C}$, et à $55^{\circ} \mathrm{C}$, température optimale d'action, l'activité résiduelle n'est plus que 15 à 25 p. 100 de l'activité initiale. Les préparations enzymatiques isolées apparaissent donc beaucoup plus sensibles à l'action des températures élevées que lorsqu'elles sont en présence du substrat.

Le chauffage à $60^{\circ} \mathrm{C}$ entraîne une dénaturation rapide du système protéolytique. Après $5 \mathrm{mn}$, on ne retrouve que 15 à $20 \mathrm{p} .100 \mathrm{de}$ l'activité initiale. La perte d'activité devient ensuite plus lente, et les courbes de stabilité présentent ainsi deux pentes bien distinctes (fig. $1 \mathrm{~d}$ ).

\section{II.2. Activité protéolytique intracellulaire}

L'activité maximale des différentes souches se situe dans la zone de $\mathrm{pH}$ 5,5-6,0. La souche G. 36 présente un optimum légèrement marqué à $\mathrm{pH} 5,5$, les autres souches montrent une activité optimale à pH 5,5-6,0 (fig. 3 a).

La température optimale d'activité se situe à $50^{\circ} \mathrm{C}-55^{\circ} \mathrm{C}$ (fig. $3 \mathrm{~b}$ ). A $40^{\circ} \mathrm{C}$ l'activité est proche de 45 p. 100 de l'activité maximale. A $60^{\circ} \mathrm{C}$ des pertes d'activité de 20 à 50 p. 100 sont observées.

L'activité intracellulaire présente une stabilité maximale à $\mathrm{pH} 6,0$; à $\mathrm{pH} 4,0$ et $\mathrm{pH} \mathrm{8,0,} \mathrm{l'activité} \mathrm{résiduelle} \mathrm{est} \mathrm{égale} \mathrm{à} 40-50 \mathrm{p}, 100$ de l'activité résiduelle maximale (fig. $3 \mathrm{c}$ ). Un chauffage de $15 \mathrm{mn}$ à $60^{\circ} \mathrm{C}$ réduit l'activité à $15-20$ p. 100 de l'activité initiale (fig. $3 \mathrm{~d}$ ).

\section{II.3. Recherche d'une enzyme active à pH faible}

Les souches G. 36, G. 819 et D. 72 ont été cultivées sur milieu Czapek-trypticase tamponné à pH 4,1 à l'aide d'un tampon succinate $0,2 \mathrm{M}$ et maintenu à ce $\mathrm{pH}$ pendant la durée de la culture $(11 \mathrm{j})$ par additions d'acide succinique. Les activités protéolytiques extra et 

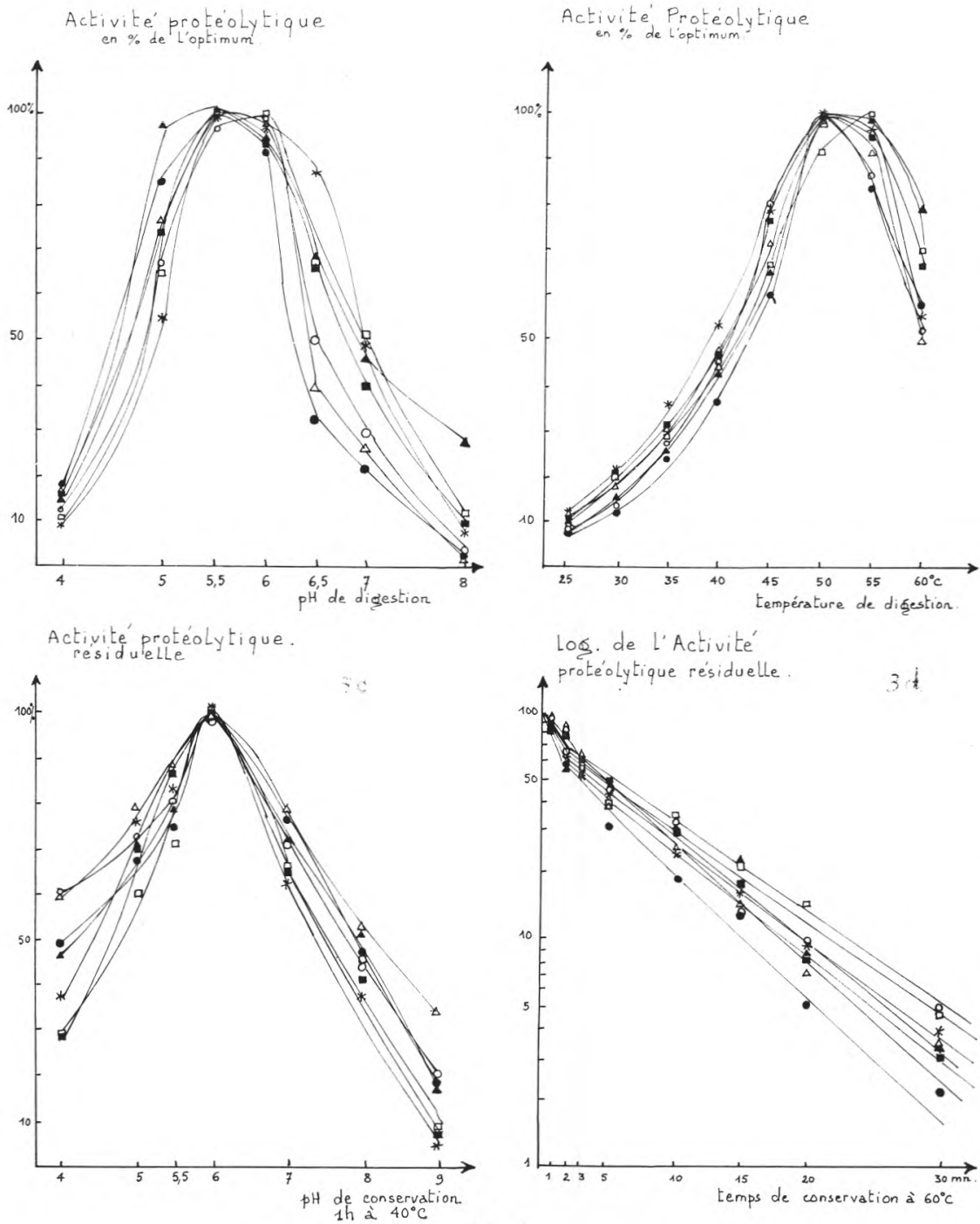

fig. 3

Caractères de l'activité protéolytique intracellulaire

$3 \mathrm{a}$ : Influence du $\mathrm{pH}$ sur l'activité.

$3 \mathrm{~b}$ : Influence de la température.

$3 \mathrm{c}$ : Influence du $\mathrm{pH}$ sur la stabilité.

$3 \mathrm{~d}$ : Stabilité thermique.

Légendes : (voir figures $1 \mathrm{a}, 1 \mathrm{~b}, 1 \mathrm{c}$ et $1 \mathrm{~d}$ ).
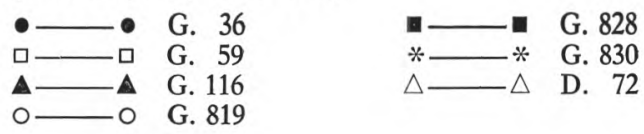
intracellulaires ont été déterminées sur les substrats caséine et hémoglobine aux $\mathrm{pH} 3,4,5$ et 6 , avec une digestion de $1 \mathrm{~h}$ à $40^{\circ} \mathrm{C}$.

Quels que soient le substrat et le $\mathrm{pH}$ de digestion, l'activité extracellulaire des trois souches est très faible. Une activité intracellulaire, plus importante avec la caséine, est mise en évidence; mais cette activité présente un optimum dans la zone de $\mathrm{pH}$ 5-6. Chez les espèces $G$. candidum et $G$. gracile il ne semble donc pas y avoir production d'une protéase acide.

\section{CONCLUSION}

Les systèmes protéolytiques des diverses souches de G. candidum présentent les mêmes caractères et il ne semble pas y avoir de différence notable entre les activités des deux espèces $G$. candidum et G. gracile.

Les activités extracellulaires et intracellulaires des souches étudiées ont des caractères très proches : $\mathrm{pH}$ optimal et température optimale d'action sont pratiquement identiques. C'est seulement au niveau de la stabilité en fonction du $\mathrm{pH}$ et de la stabilité thermique que des différences sont constatées mais on peut remarquer que l'allure générale des courbes est comparable.

Les courbes de stabilité thermique suggèrent l'existence de deux activités. Les premiers essais de purification réalisés sur des préparations extracellulaires par filtration sur gel et chromatographie sur DEAE cellulose confirment la présence de deux enzymes aptes à manifester leur action dans les pâtes fromagères.

\section{Ré s u m é}

Les caractères du système protéolytique de Geotrichum candidum Link. ont été étudiés sur six souches d'activités différentes. Ils ont été comparés à ceux d'une souche appartenant à l'espèce Geotrichum gracile.

Les préparations enzymatiques extracellulaires ont un $\mathrm{pH}$ optimal situé dans la zone 5,5-6,0, une température optimale de $55^{\circ} \mathrm{C}$; celles d'origine intracellulaire présentent également une activité maximale à $\mathrm{pH}$ 5,5-6,0, une température optimale de $50^{\circ} \mathrm{C}-55^{\circ} \mathrm{C}$. Les deux préparations diffèrent par leur stabilité ; les préparations extracellulaires sont plus rapidement inactivées à $\mathrm{pH} 4,0$ et plus thermolabiles.

L'allure des courbes de stabilité thermique suggère l'existence de deux enzymes ; il ne semble pas y avoir, cependant, production d'une protéase acide. 


\section{S u $\mathrm{m} \mathrm{m}$ a r y}

The characteristics of the extra and intracellular proteolytic systems of six strains of Geotrichum candidum which exhibited different proteolytic levels have been studied and compared with those of a strain of $G$. gracile.

The maximum casein hydrolysis by the extracellular system of all strains occurs over a $\mathrm{pH}$ range of 5,5 to 6,0 at an optimum temperature of $55^{\circ} \mathrm{C}$; for the intracellular system, the optimum conditions are also a $\mathrm{pH}$ range of 5,5 to 6,0 and a temperature range of $50^{\circ} \mathrm{C}$ to $55^{\circ} \mathrm{C}$. The stabilities of the two systems are different, the extracellular one being more thermolabile and more rapidly inactivated at $\mathrm{pH} 4,0$.

The plot of thermic stability curves suggests the presence of two enzyms ; however, no acid protease has been found.

Reçu pour publication en mars 1976.

\section{Bibliographie}

ANSON (M. L.) (1938). - The estimation of pepsin, papaïn, and cathepsin with hemoglobulin. J. Gen. Physiol., 73, 627.

Carini (S.), Volonterio (G.), Bozzolati (M.), Galli (A.) (1970). - Caseinolosi e lipolisi di Mucor, Penicillium e Geotrichum. Latte, 44, (12), 867-873.

DaLE (G.) (1972). - Moisissures et levures de la flore du fromage de St-Nectaire. Revue Lait. Frse "Industr. Lait. ", 296, 199-203.

Dale (G.), Guillot (J.) (1971). - Contribution à l'étude de la microflore du fromage de St-Nectaire et de la physiologie de Geotrichum Candidum Link. Société Biologique de Clermont-Ferrand, 309-316.

Delespaul (G.), Gueguen (Micheline), Lenoir (J.) (1973). - La flore fongique superficielle des fromages de St-Nectaire et de Tome de Savoie. Son évolution au cours de l'affinage Revue Lait. Frse "Industr. Lait. ", 313, 715-729.

DluzewsKi (M.) (1963). - Lypolytic properties of Oospora lactis. Bull. Acad. Sci. Ser. Sci. Biol., 11, (5), 227-230.

Franzke (Cl.), Kroll (J.), Petzold (R.) (1973). - Studien zur Glyceridstruktur von Fetten 6. Mitt. Untersuchungen und Synthetisierten Triglyceriden zur Fettsäuresperifität der Lipase von Geotrichum candidum. Nahrung, 17, (2), 171-184.

Gueguen (Micheline), Delespaul (G.), Lenoir (J.) (1974). - La flore fongique superficielle des fromages de St-Nectaire et de Tome de Savoie. II. Ses conditions de développement. Revue Lait. Frse "Industr. Lait. ", 325, 1-11.

Gueguen (Micheline), LenoIR (J.) (1975 a). - Aptitude de l'espèce Geotrichum candidum à la production d'enzymes protéolytiques. Le Lait, t. 55, (543-544), 145-162.

GuEguen (Micheline), LenoIR (J.) (1975 b). - Aptitude de l'espèce Geotrichum candidum à la production d'enzymes protéolytiques. Note complémentaire. Le Lait, t. 55, (549-550), 621-629. 
Hurel (C.), Mocouot (G.) (1947). - Etude comparée des fromages de Livarot et Pont-l'Evêque. Le Lait, 27, 131-141.

Nelson (W. O.) (1952). - Some r.taracteristics of the lipase of Geotrichum candi. dum. J. Dairy Sci., 35, 455-462.

NelsoN (W. O.) (1953). - Nutritional factors affecting growth and production of lipase by Geotrichum candidum. J. Dairy Sci., 36, 143-151.

SANSONETTI (Françoise) (1930). - Sur les flores bactériennes et fongiques des caillés lactiques. Thèse Doctorat en Pharmacie, Paris. Le Lait, 10, (96-100).

THom (C.), RAPER (K. B.) (1945). - A manual of the aspergilli. The Williams and Wilking Company Ed., Baltimore (U.S.), p. 373.

Tsujisaka (Y.), Iwai (M.), Tominaga (Y.) (1973). - Purification, crystallization and some properties of lipase from Geotrichum candidum Link. Agr. Biol. Chem., 37, (6), 1457-1464. 